\title{
Device-In-Use Domain
}

National Cancer Institute

\section{Source}

National Cancer Institute. Device-In-Use Domain. NCI Thesaurus. Code C102622.

A subject domain utilized for the submission of information encompassing and representing data, vocabulary or records related to device-in-use. 